\title{
Prediction of patients requiring intensive care for COVID-19: development and validation of an integer- based score using data from Centers for Disease Control and Prevention of South Korea.
}

\section{JoonNyung Heo}

Armed Forces Medical Command https://orcid.org/0000-0001-6287-6348

\section{Deokjae Han}

Division of Pulmonary and Critical Care Medicine, Department of Internal Medicine, Armed Forces Capital Hospital

Hyung-Jun Kim

Division of Pulmonary and Critical Care Medicine, Department of Internal Medicine, Armed Forces Capital Hospital

\section{Daehyun Kim}

Department of Periodontology, The Armed Forces Capital Hospital

\section{Yeon-Kyeng Lee}

Division of Chronic Disease Control, Korea Center for Disease Control and Prevention

\section{Dosang Lim}

Division of Chronic Disease Control, Korea Center for Disease Control and Prevention

\section{Sung Ok Hong}

Division of Chronic Disease Control, Korea Center for Disease Control and Prevention

\section{Mi-Jin Park}

Division of Chronic Disease Control, Korea Center for Disease Control and Prevention

\section{Beomman $\mathrm{Ha}$}

Armed Forces Medical Command

Woong Seog ( $\sim$ drscopy@naver.com )

Armed Forces Medical Command https://orcid.org/0000-0001-5016-6103

\section{Research Article}

Keywords: COVID-19, Critical care, Prognosis

Posted Date: October 6th, 2020

DOl: https://doi.org/10.21203/rs.3.rs-87981/v1 
License: (c) (i) This work is licensed under a Creative Commons Attribution 4.0 International License. Read Full License

Version of Record: A version of this preprint was published at Journal of Intensive Care on January 29th, 2021. See the published version at https://doi.org/10.1186/s40560-021-00527-x. 


\section{Abstract}

Background Unavailability or saturation of the intensive care unit may be associated with the fatality of COVID-19. Prioritizing the patients for hospitalization and intensive care may be critical for reducing the fatality of COVID-19. This study aimed to develop and validate a new integer-based scoring system for predicting patients with COVID-19 requiring intensive care, using only the predictors available upon triage.

Methods This is a retrospective study using cohort data from the Korean Centers for Disease Control and Prevention that included all admitted patients with COVID-19 between January 19 and June 3, 2020, in South Korea. The primary outcome was patients requiring intensive care defined as actual admission to the intensive care unit; at any time use of an extracorporeal life support device, mechanical ventilation, or vasopressors; death. Patients admitted until March 20 were included for the training dataset to develop the prediction models and externally validated for the patients admitted afterward. Two logistic regression models were developed with different predictors and the predictive performance was compared: one with patient-provided variables and the other with added radiologic and laboratory variables. An integer-based scoring system was developed based on the developed logistic regression model.

Results A total of 5,193 patients were considered, with 4,663 patients included after excluding patients with age under 18 or insufficient data. For the training dataset, 3,238 patients were included. Of the included patients, 444 (9.5\%) patients required intensive care. The model developed with only the clinical variables showed an area under the curve of 0.884 for the validation set. The performance did not differ when radiologic and laboratory variables were added. Seven variables were selected for developing an integer-based scoring system: age, sex, initial body temperature, dyspnea, hemoptysis, history of chronic kidney disease, and activities of daily living. The area under the curve of the scoring system was 0.880 .

Conclusions An integer-based scoring system was developed for predicting patients with COVID-19 requiring intensive care, with high performance. This system may aid decision support for prioritizing the patient for hospitalization and intensive care, particularly in a situation with limited medical resources.

\section{Background}

COVID-19 is a pandemic with over 12 million confirmed cases worldwide as of July 10, 2020. Death from the disease exceeded 500,000. Death rates differ among countries and even among cities, and medical resource availability may be a major factor of the differences.[1] The fast spread of the virus is causing excessive stress on the public health systems.[2] Efforts are being made to alleviate this stress by increasing medical supply,[3] but it may not be enough for an overwhelming outbreak, especially for situations with limited medical resources. 
COVID-19 is mild or asymptomatic in about $80-90 \%$ of cases.[4] Rates for the cases requiring intensive care are low, with $10-20 \%$ being admitted to the intensive care unit (ICU), 3-10\% requiring intubation, and 2-5\% dead.[5] Severe patients show respiratory failure, pneumonia, multi-organ failure, and shock, which require care in intensive care facilities. In those patients, care in ICU is critical for survival. While the mortality rate is variable among countries, unavailability or saturation of ICU is one of the crucial factors that affect the mortality.[4] ICU capacity differs among countries, lower in lower-middle-income countries. [6] Within the ICU, patients with COVID-19 should be admitted to an airborne infection isolation room to protect other admitted patients and medical staff from the transmission of COVID-19. As such, there are concerns about the limited availability of ICU facilities for patients with COVID-19.

While it is uncertain how many and which patients with COVID-19 need hospitalization and care in ICU, many patients with mild symptoms are often hospitalized due to a fear of aggravation and the necessity of quarantine. This may further exhaust medical resources including the availability of hospital beds under resource-limiting conditions. In this context, prioritizing patients with COVID-19 for care in a professional medical facility, especially for the ICU, may help reduce the mortality rate in COVID-19 epidemic hotspots.

The course of COVID-19 is variable. The median time from symptom onset to severe hypoxemia and ICU admission is approximately 7-12 days.[7] Although the rates of patients needing ICU care is currently unknown, $6.1 \%$ were classified as critical and $13.8 \%$ as severe.[8] However, it is often unpredictable who will need ICU care at the early stage of disease or presentation to the hospital.[7] Predicting the patients with COVID-19 at risk of death or needing ICU care may help prioritize the hospitalization of the patient at triage.

In this study, we aimed to develop and validate a model for predicting admission to ICU at presentation to the hospital using data from a nation-wide cohort of patients with COVID-19 in South Korea.

\section{Methods}

\section{Study population}

This was a retrospective study using cohort data that included all patients with COVID-19 in South Korea from 100 hospitals. The cohort was developed and managed by the Korean Centers for Disease Control and Prevention (KCDC). Patients with laboratory-confirmed COVID-19 were either admitted to a hospital or a community treatment center according to regional risk stratification and triage system.[9] KCDC mandated the hospitals to register their patients' data to the cohort. Among the admitted patients, those who died or confirmed free of disease after management and released from quarantine from January 25 , 
2020 to June 3, 2020 were included in this study. Patients who were admitted until March 20, 2020 were used for the training dataset, and temporal external validation was performed on the patients admitted afterward. All patients were included for this study, except for those under 18 years old or those with incomplete data.

\section{Data and variables}

Variables used for developing the outcome prediction model included those for the outcome, demographics, medical history, clinical symptoms and signs, imaging findings, and laboratory results. Admission to ICU, use of extracorporeal life support (ECLS), mechanical ventilation, or vasopressors, and death were included to derive the primary outcome. Demographic variables included age and sex. Medical history included pregnancy, diabetes, heart failure, hypertension, chronic cardiac disease, asthma, chronic obstructive pulmonary disease, chronic kidney disease, cancer, chronic liver disease, chronic neurologic disorders, chronic hematologic disorders, human immunodeficiency virus infection, dementia, smoking status, and activities of daily living (ADL) scale. Clinical variables included initial body temperature, cough, sputum, hemoptysis, sore throat, rhinorrhea, chest discomfort, myalgia, fatigue, and dyspnea. The only variable included from the imaging results was a binary value of whether there was any infiltration shown in the initial chest X-ray. Laboratory findings included counts of white blood cell, lymphocyte, and platelet; and levels of hemoglobin, hematocrit, albumin, aspartate aminotransferase, alanine aminotransferase, blood urea nitrogen, and creatinine. Initial values that were acquired for the patients were used.

\section{Outcomes}

The primary outcome was patients requiring intensive care defined as actual admission to the ICU; at any time use of an ECLS device, mechanical ventilation, or vasopressors; death. Death was defined when the patient died during the follow-up period.

\section{Statistical analysis}

Descriptive statistics were performed for all variables. Two logistic regression models were developed to assess the importance of input variables. These two models had the same prediction target as the patients requiring ICU care, but with different predictors. The first model was built only with variables that can be acquired during triage, that can easily be provided by the patient, including the patient's demographics, medical history, clinical symptoms, and body temperature. The other model used imaging findings and radiologic results in addition to all variables from the first model. The area under the curve 
(AUC) was calculated for the training set and the validation set to assess the predictive accuracy of the model.

From the two logistic regression models, variables that were statistically significant during the stepwise backward elimination were used to build the integer-based scoring model. Coefficients from the first model, which used only the variables that can be provided by the patient, were multiplied by 4 and rounded to the nearest integer to generate an integer-based scoring system. The overall score was calculated as the sum of the scores. The scoring system was applied to the validation dataset to acquire the accuracy of the model. All $P$ values were 2 -sided, and $P<0.05$ was considered statistically significant. Statistical analysis was performed using R, and packages MASS and caret were used.[10-12]

\section{Results}

A total of 5,193 patients with confirmed COVID-19 from 100 centers was registered to the cohort during the study period. Patients under 18 were excluded (117 patients), and those with insufficient data were excluded (413 patients), leaving 4,663 patients for analysis. From the included patients, 444 (9.5\%) patients required ICU care, $213(4.6 \%)$ patients were actually admitted to ICU, and 217 (4.7\%) patients died. A total of 3,238 patients were admitted before March 20, 2020 and used to develop the prediction models. Patients admitted after were used to validate the developed model, and 1,425 patients were included (Table 1).

Table 1. Comparison between the training set and the validation set 


\section{Total $(n=4663) \quad$ Training set \\ $(n=3238)$}

Validation set

$(n=1425)$
$P$
Value

Demographic

Age

Sex, Male

Symptoms and signs temperature

Initial body

Cough

Sputum

Hemoptysis

Sore throat

Rhinorrhea

Chest pain

Myalgia

Arthralgia

Lethargic

Dyspnea

Headache

Nausea, vomiting

Diarrhea

Medical history

Pregnancy

Diabetes

Heart failure

Hypertension

disease

Chronic cardiac

Asthma

COPD

Chronic kidney
$55.0[37.0 ; 67.0] \quad 56.0[41.0 ; 68.0]$

$1841(39.5 \%)$
$53.0[30.0 ; 66.0]$

$659(46.2 \%)$
$36.8[36.5 ; 37.3] \quad 36.9[36.5 ; 37.3]$

$1944(41.7 \%) \quad 1523(47.0 \%)$

$1344(28.8 \%) \quad 1049(32.4 \%)$

$26(0.6 \%)$

$682(14.6 \%)$

$440(9.4 \%)$

$351(7.5 \%)$

$733(15.7 \%)$

$18(0.4 \%)$

$179(3.8 \%)$

$632(13.6 \%)$

$773(16.6 \%)$

$216(4.6 \%)$

$400(8.6 \%)$
$23(0.7 \%)$

514 (15.9\%)

$334(10.3 \%)$

$294(9.1 \%)$

$583(18.0 \%)$

$16(0.5 \%)$

$143(4.4 \%)$

$528(16.3 \%)$

$612(18.9 \%)$

171 (5.3\%)

333 (10.3\%)
$36.8[36.5 ; 37.2]$

0.001

$421(29.5 \%)$

$<0.001$

295 (20.7\%)

$<0.001$

0.058

$3(0.2 \%)$

$<0.001$

168 (11.8\%)

0.002

106 (7.4\%)

$<0.001$

57 (4.0\%)

$<0.001$

150 (10.5\%)

0.124

2 (0.1\%)

0.003

36 (2.5\%)

$<0.001$

104 (7.3\%)

$<0.001$

$161(11.3 \%)$

0.002

45 (3.2\%)

$<0.001$

67 (4.7\%)

.

$17(0.4 \%)$

$12(0.4 \%)$

$5(0.4 \%)$

1.000

$510(15.8 \%)$

$194(13.6 \%)$

0.067

$66(1.4 \%)$

$40(1.2 \%)$

$26(1.8 \%)$

0.151

$1183(25.4 \%)$

$847(26.2 \%)$

$336(23.6 \%)$

0.068

$188(4.0 \%)$

$138(4.3 \%)$

50 (3.5\%)

0.261

118 (2.5\%)

93 (2.9\%)

25 (1.8\%)

0.033

40 (0.9\%)

$34(1.1 \%)$

$6(0.4 \%)$

0.048

$56(1.2 \%)$

$45(1.4 \%)$

$11(0.8 \%)$

0.101 
disorder

$\begin{array}{lllll}\text { Cancer } & 154(3.3 \%) & 111(3.4 \%) & 43(3.0 \%) & 0.526\end{array}$
disorder

Chronic hematologic $\quad 34(0.7 \%)$

HIV infection

Autoimmune disease

Dementia

Smoking

(Never smoker)

(Ex-smoker)

(Current smoker)

ADL

(Normal)

(Partially dependent)

(Totally dependent)

Imaging and laboratory

findings

$g / d L$

Chest X-ray infiltration

Hemoglobin level,

Platelet count, $10^{3} / \mu \mathrm{L}$

224.0

[176.0;280.0]

227.0

[178.0;283.0]

WBC count, $10^{3} / \mu \mathrm{L}$

Lymphocyte, \%

Hematocrit, \%

AST level, IU/L

ALT level, IU/L

Albumin level, $\mathrm{g} / \mathrm{dL}$

BUN level, mg/dL
$5.7[4.4 ; 7.1]$

$28.7[20.9 ; 36.2]$

$39.1[36.2 ; 42.3]$

$24.0[19.0 ; 33.0]$

$20.0[14.0 ; 31.0]$

$4.2[3.8 ; 4.5]$

$12.3[10.0 ; 15.8] \quad 12.5[10.0 ; 16.0]$

$5.7[4.5 ; 7.2]$

$28.7[20.9 ; 36.1]$

$39.0[36.1 ; 42.1]$

$25.0[20.0 ; 34.0]$

$20.0[14.0 ; 31.0]$

$4.1[3.8 ; 4.4]$
$4.3[3.9 ; 4.6]$

$12.0[9.7 ; 15.0]$
0.897

0.091

$18(1.3 \%)$

$7(0.5 \%)$

0.280

$2(0.1 \%)$

0.856

7 (0.5\%)

0.380

148 (10.4\%)

$<0.001$

$<0.001$

1246 (87.4\%)

$37(2.6 \%)$

$142(10.0 \%)$

$<0.001$

1127 (79.1\%)

164 (11.5\%)

134 (9.4\%) 
$\mathrm{mg} / \mathrm{dL}$

$\begin{array}{lllll}\text { Creatinine level, } & 0.7[0.6 ; 0.9] & 0.7[0.6 ; 0.9] & 0.8[0.6 ; 0.9] & 0.342\end{array}$

Outcomes

\begin{tabular}{|cllll|}
\hline Requiring ICU & $444(9.5 \%)$ & $320(9.9 \%)$ & $124(8.7 \%)$ & 0.226 \\
\hline $\begin{array}{c}\text { Admission to ICU } \\
\begin{array}{c}\text { Mechanical } \\
\text { ventilation }\end{array}\end{array}$ & $213(4.6 \%)$ & $113(3.5 \%)$ & $70(5.0 \%)$ & 0.016 \\
\hline ECLS & $49(1.1 \%)$ & $35(1.1 \%)$ & $14(1.0 \%)$ & 0.697 \\
\hline $\begin{array}{c}\text { Vasopressor } \\
\text { treatment }\end{array}$ & $28(0.6 \%)$ & $22(0.7 \%)$ & $6(0.4 \%)$ & 0.397 \\
\hline Death & $118(2.5 \%)$ & $83(2.6 \%)$ & $35(2.5 \%)$ & 0.182 \\
\hline
\end{tabular}

$A D L$ activities of daily living, $A S T$ aspartate aminotransferase, $A L T$ alanine aminotransferase, $B U N$ blood urea nitrogen, $C O P D$ chronic obstructive pulmonary disease, ECLS extracorporeal life support, $H I V$ human immunodeficiency virus, $I C U$ intensive care unit, $W B C$ white blood cell.

Values are number (\%) or median [interquartile range].

\section{Accuracy of the two prediction models}

Multivariate logistic regression models were built with stepwise backward elimination of insignificant variables. The first model, developed with only the variables that a patient can provide showed an AUC of 0.869 [95\% $\mathrm{Cl} 0.845-0.893]$ for the training set, and 0.884 [95\% $\mathrm{Cl} 0.852-0.916]$ for the validation set. The second model, which included the findings from the initial chest X-ray and the laboratory results in addition to the first model, showed a slightly increased accuracy (AUC 0.899 [95\% Cl 0.877-0.920] for the training set and AUC 0.897 [95\% $\mathrm{Cl} 0.865-0.928]$ for the validation set) but was not significant $(P=$ 0.169) (Fig 1).

\section{Integer-based scoring system}

Using the multivariate logistic regression analysis results from the first model, a total of seven variables were selected as significant predictors for the patients requiring ICU care: sex, age, initial body temperature on admission, dyspnea, hemoptysis, history of chronic kidney disease, and ADL scale (Table 2). The mean variable inflation factor was 1.08 (range 1.01-1.24), showing low collinearity. The coefficients of the model were multiplied by 4 and rounded to the nearest integer, to form an integerbased scoring system to predict patients with COVID-19 requiring ICU care (COVIC score). Continuous 
variables (age and initial body temperature) were categorized, with the median value used to calculate the score point for the category. For the continuous variables, the score for each category was subtracted by the score of the lowest category, for ease of calculation. As a result, the score of the lowest category was set to zero for age and temperature (Table 3 ).

Table 2. Predictors of patients requiring intensive care unit care in multivariate logistic regression.

\begin{tabular}{|c|c|c|c|c|}
\hline Variables & Odds Ratio & Lower $95 \% \mathrm{Cl}$ & Upper $95 \% \mathrm{Cl}$ & Std Error \\
\hline Age $^{*}$ & 1.039 & 1.029 & 1.050 & 0.005 \\
\hline Sex, female & 0.369 & 0.280 & 0.487 & 0.141 \\
\hline Initial body temperature on admission* & 1.506 & 1.234 & 1.835 & 0.101 \\
\hline Hemoptysis & 5.371 & 1.737 & 15.539 & 0.554 \\
\hline Dyspnea & 5.124 & 3.846 & 6.834 & 0.147 \\
\hline Chronic kidney disease & 4.119 & 1.921 & 8.714 & 0.385 \\
\hline ADL score $1^{\star \star}$ & 4.113 & 2.751 & 6.122 & 0.204 \\
\hline ADL score $2^{* *}$ & 8.753 & 5.771 & 13.285 & 0.212 \\
\hline
\end{tabular}

Cl confidence interval, Std Errorstandard error, $A D L$ activities of daily living.

${ }^{*}$ Continuous variables.

${ }^{*} \mathrm{ADL}$ score of 1 represents partially dependent, and 2 represents totally dependent on others.

Table 3. Scoring system to predict COVID-19 patients requiring ICU care (COVIC score). 


\begin{tabular}{|c|c|c|}
\hline Variables & & Score \\
\hline \multirow[t]{6}{*}{ Age, years } & 18 - less than 30 & 0 \\
\hline & 30 - less than 40 & 1 \\
\hline & 40 - less than 50 & 3 \\
\hline & 50 - less than 60 & 4 \\
\hline & 60 - less than 70 & 6 \\
\hline & $\geq 70$ & 7 \\
\hline Male sex & & 4 \\
\hline \multirow[t]{5}{*}{ Initial body temperature on admission, ${ }^{\circ} \mathrm{C}$} & $<37.0$ & 0 \\
\hline & 37.0 - less than 37.5 & 1 \\
\hline & 37.5 - less than 38.0 & 2 \\
\hline & 38.0 - less than 39 & 3 \\
\hline & $\geq 39.0$ & 4 \\
\hline Hemoptysis & & 7 \\
\hline Dyspnea & & 7 \\
\hline Chronic kidney disease & & 6 \\
\hline \multirow[t]{2}{*}{ Activities of daily living * } & 1 - Partially dependent & 6 \\
\hline & 2 - Totally dependent & 9 \\
\hline
\end{tabular}

${ }^{*}$ Activities of daily living score of 1 represents partially dependent, and 2 represents totally dependent on others.

The performance of the newly developed scoring system was externally validated. The receiver operating characteristic curve was drawn with an AUC of 0.880 [95\% Cl, 0.847-0.912] (Fig 2). The association between the integer-based score and the probability of the patient requiring ICU care for the total dataset is presented in Fig 3. The Hosmer-Lemeshow test was performed with the Chi-squared value of 8.866 and the $P$-value of 0.35 , suggesting goodness of fit (Fig 4 ).

For better accessibility and ease of use, a web-based application was developed (Fig 5). The application can be accessed at http://covic.docl.org. 


\section{Discussion}

This study presents and validates an integer-based scoring system for prediction of patients with COVID19 requiring ICU care (COVIC score), with excellent prediction performance. The scoring system consists of eight variables: sex, age, initial body temperature, hemoptysis, dyspnea, history of chronic kidney disease, and ADL scale.

Previous studies have shown that male sex, old age, and comorbidities including active cancer, coronary artery disease, liver and kidney dysfunctions, chronic obstructive pulmonary disease, diabetes, hypercholesterolemia were factors associated with mortality among patients with COVID-19 who were admitted to ICU.[13, 14] These findings and ours suggest that patients with COVID-19 may have an increased risk of grave outcome or need intensive care when they are old, male, and have comorbidities. Our findings suggest that additional presence of some symptoms and signs such as fever, hemoptysis, dyspnea, and dependent activities in daily living should be considered as a clue that patients may need intensive care during the course.

There were previous studies to predict patient outcomes for COVID-19.[15-19] Mortality risk, hospital stay, and progression to severe state were the primary outcome for the prediction models.[15-26] One of the largest cases included were 1,590 cases from a national retrospective cohort from China.[19] In that study, the scoring system included 10 variables, which were chest radiographic abnormality, age, hemoptysis, dyspnea, unconsciousness, number of comorbidities, cancer history, and laboratory values. Some of them are similar to variables in our scoring system but included those that can only be assessed after professional care, such as imaging results and laboratory values.

In this study, logistic regression analysis showed that additional variables including chest X-ray findings and laboratory results may not significantly increase the performance of the prediction for patients requiring ICU care. The variables that constitute the developed scoring system from this study do not require professional medical assessment but can be provided by the patient. As such, this scoring system may help to prioritize the patient for hospitalization at triage.

The scoring system in this study can be applied to wider usage. For instance, when the diagnostic capacity or hospital beds are insufficient to meet the demand of the patients with COVID-19, prioritizing the patient for hospitalization may be critical. If the patient is hospitalized on a first-come-first-served basis, then patients who may require ICU care during the course, but who is slower in arrival to the clinics, may not be able to receive proper management. In addition, this scoring system may help the paramedics 
transfer the patients suspected of COVID-19 to the appropriate hospital. Even for the undiagnosed patients, if needed, the feature that can be self-calculated by the patient may be useful.

In this study, the primary outcome, patients requiring ICU care, was defined as actual admission to the ICU; at any time use of an ECLS device, mechanical ventilation, or vasopressors; death. Rather than choosing the patients admitted to the ICU, the sum of the patients listed above was chosen as the primary outcome since there were many hospitals with negative pressure quarantine rooms that substituted the ICU in case of saturated or lacked ICU in South Korea. In addition, the use of an ECLS device, mechanical ventilation, or vasopressors is an ample indicator of needing intensive care.

The dataset used for this study was provided by the KCDC, with all admitted cases in South Korea subject to registration for the cohort. The remaining patients with COVID-19 were managed in a community treatment center. As a result, $56 \%(5,193$ out of 9,306) of all South Korean patients with COVID-19 during the study period were analyzed for this study. Fortunately, the hospital beds are relatively sufficient in South Korea, and the maximum number of confirmed cases per day being 1,062 cases (March 1, 2020). This ensured that the patients residing outside of the hospitals were neither critical nor needed hospitalization. For instance, anyone older than 65 years or with a chronic comorbidity such as diabetes were mandated to be admitted to a hospital by KCDC guidelines,[27] No confirmed patients were residing at home during the study period. As a result, there should be a very low risk of selection bias of the cohort that this study is based on.

This study has several limitations. While there are discrepancies in medical infrastructure between countries, this study was conducted in one country with ethnic homogeneity $(98.2 \%$ were of Korean ethnicity). In addition, this was a retrospective study. Therefore, external validation for a different nation or ethnicity will be needed. About $8 \%$ of patients were excluded from developing the models due to insufficient data or young age.

\section{Conclusions}

An integer-based scoring system was developed for the prediction of patients with COVID-19 requiring ICU care, with high performance. The developed model may be able to aid decision support for prioritizing the patient for hospitalization, especially for the circumstances where medical resources are limited.

\section{List Of Abbreviations}


ADL: activities of daily living; AUC: area under the curve; ECLS: extracorporeal life support; ICU: intensive care unit; KCDC: Korean Centers for Disease Control and Prevention

\section{Declarations}

\section{Ethics approval and consent to participate}

This study was approved by the Institutional Review Board of Armed Forces Medical Command with a waiver of informed consent due to the retrospective nature of the study.

\section{Consent for publication}

Not applicable.

\section{Availability of data and materials}

The datasets generated and/or analyzed during the current study are available in the Korean Centers for Disease Control and Prevention's COVID-19 Clinical Epidemiologic Information Repository, https://www.cdc.go.kr/board/board.es?mid=a20504000000\&bid=0014.

\section{Competing interests}

The authors declare that they have no competing interests.

\section{Funding}

None.

\section{Authors' contributions}

$\mathrm{JH}$ conceptualized the design of the work, analyzed and interpreted the data, and drafted the manuscript.

$\mathrm{DH}$ and $\mathrm{HK}$ contributed to the design of the work and interpreted the analysis results.

DK contributed to the design of the work.

$\mathrm{YL}, \mathrm{DL}, \mathrm{SH}$, and MP contributed to the acquisition and pre-processing of the data. 
$\mathrm{BH}$ and WS contributed to the acquisition of data, interpreted the analysis results, and substantially revised the final manuscript.

\section{Acknowledgments}

We acknowledge all the health-care workers involved in the diagnosis and treatment of COVID-19 patients in South Korea. We thank the Central Disease Control Headquarters, National Medical Center and the Health Information Manager in 100 hospitals for their effort in collecting the medical records.

\section{References}

1. Ji Y, Ma Z, Peppelenbosch MP, Pan Q. Potential association between COVID-19 mortality and healthcare resource availability. The Lancet Global Health. 2020;8:e480.

2. Wang C, Horby PW, Hayden FG, Gao GF. A novel coronavirus outbreak of global health concern. The Lancet. 2020;395:470-3. doi:10.1016/S0140-6736(20)30185-9.

3. Ranney ML, Griffeth V, Jha AK. Critical Supply Shortages - The Need for Ventilators and Personal Protective Equipment during the Covid-19 Pandemic. The New England journal of medicine. 2020;382:e41. doi:10.1056/NEJMp2006141.

4. Pascarella G, Strumia A, Piliego C, Bruno F, del Buono R, Costa F, et al. COVID-19 diagnosis and management: a comprehensive review. Journal of Internal Medicine. 2020; March:1-15.

5. Guan W, Ni Z, Hu Y, Liang W, Ou C, He J, et al. Clinical characteristics of coronavirus disease 2019 in China. New England Journal of Medicine. 2020;382:1708-20.

6. Phua J, Weng L, Ling L, Egi M, Lim CM, Divatia JV, et al. Intensive care management of coronavirus disease 2019 (COVID-19): challenges and recommendations. The Lancet Respiratory Medicine. 2020;8:506-17. doi:10.1016/S2213-2600(20)30161-2.

7. Phua J, Weng L, Ling L, Egi M, Lim CM, Divatia JV, et al. Intensive care management of coronavirus disease 2019 (COVID-19): challenges and recommendations. The Lancet Respiratory Medicine. 2020;8:506-17. doi:10.1016/S2213-2600(20)30161-2.

8. Report of the WHO-China Joint Mission on Coronavirus Disease 2019 (COVID-19). https://www.who.int/publications/i/item/report-of-the-who-china-joint-mission-on-coronavirusdisease-2019-(covid-19). Accessed 15 Jul 2020.

9. Kim J-H, An JA-R, Min P, Bitton A, Gawande AA. How South Korea Responded to the Covid-19 Outbreak in Daegu. NEJM Catalyst. 2020;1. doi:10.1056/cat.20.0159.

10. Kuhn M. caret: Classification and Regression Training. 2020. https://cran.rproject.org $/$ package $=$ caret.

11. Venables WN, Ripley BD. Modern Applied Statistics with S. Fourth. New York: Springer; 2002. http://www.stats.ox.ac.uk/pub/MASS4. 
12. R Core Team. R: A Language and Environment for Statistical Computing. 2020.

13. Grasselli G, Greco M, Zanella A, Albano G, Antonelli M, Bellani G, et al. Risk Factors Associated With Mortality Among Patients With COVID-19 in Intensive Care Units in Lombardy, Italy. JAMA internal medicine. 2020. doi:10.1001/jamainternmed.2020.3539.

14. Gupta S, Hayek SS, Wang W, Chan L, Mathews KS, Melamed ML, et al. Factors Associated With Death in Critically III Patients With Coronavirus Disease 2019 in the US. JAMA internal medicine. 2020. doi:10.1001/jamainternmed.2020.3596.

15. Gong J, Ou J, Qiu X, Jie Y, Chen Y, Yuan L, et al. A Tool to Early Predict Severe Corona Virus Disease 2019 (COVID-19): A Multicenter Study using the Risk Nomogram in Wuhan and Guangdong, China. Clinical Infectious Diseases. 2020.

16. Yuan M, Yin W, Tao Z, Tan W, Hu Y. Association of radiologic findings with mortality of patients infected with 2019 novel coronavirus in Wuhan, China. PLOS ONE. 2020;15:e0230548. doi:10.1371/journal.pone.0230548.

17. Yan L, Zhang H-T, Xiao Y, Wang M, Sun C, Liang J, et al. Prediction of criticality in patients with severe Covid-19 infection using three clinical features: a machine learning-based prognostic model with clinical data in Wuhan. medRxiv. 2020;:2020.02.27.20028027. doi:10.1101/2020.02.27.20028027.

18. Xie J, Hungerford D, Chen H, Abrams ST, Li S, Wang G, et al. Development and External Validation of a Prognostic Multivariable Model on Admission for Hospitalized Patients with COVID-19. SSRN Electronic Journal. 2020.

19. Liang W, Liang H, Ou L, Chen B, Chen A, Li C, et al. Development and Validation of a Clinical Risk Score to Predict the Occurrence of Critical Illness in Hospitalized Patients with COVID-19. JAMA Internal Medicine. 2020.

20. Shi Y, Yu X, Zhao H, Wang H, Zhao R, Sheng J. Host susceptibility to severe COVID-19 and establishment of a host risk score: Findings of 487 cases outside Wuhan. Critical Care. 2020;24:108. doi:10.1186/s13054-020-2833-7.

21. Bai X, Fang C, Zhou Y, Bai S, Liu Z, Xia L, et al. Predicting COVID-19 Malignant Progression with AI Techniques. SSRN Electronic Journal. 2020.

22. Qi X, Jiang Z, YU Q, Shao C, Zhang H, Yue H, et al. Machine learning-based CT radiomics model for predicting hospital stay in patients with pneumonia associated with SARS-CoV-2 infection: A multicenter study. medRxiv. 2020;:2020.02.29.20029603.

23. Lu J, Hu S, Fan R, Liu Z, Yin X, Wang Q, et al. ACP risk grade: a simple mortality index for patients with confirmed or suspected severe acute respiratory syndrome coronavirus 2 disease (COVID-19) during the early stage of outbreak in Wuhan, China. medRxiv. 2020;:2020.02.20.20025510.

24. Caramelo F, Ferreira N, Oliveiros B. Estimation of risk factors for COVID-19 mortality - preliminary results. medRxiv. 2020;19:2020.02.24.20027268. doi:10.1101/2020.02.24.20027268.

25. Williams RD, Markus AF, Yang C, Salles TD, Falconer T, Jonnagaddala J, et al. Seek COVER: Development and validation of a personalized risk calculator for COVID-19 outcomes in an international network. medRxiv. 2020;:2020.05.26.20112649. 
26. Ji D, Zhang D, Xu J, Chen Z, Yang T, Zhao P, et al. Prediction for Progression Risk in Patients with COVID-19 Pneumonia: the CALL Score. Clinical infectious diseases: an official publication of the Infectious Diseases Society of America. 2020;:ciaa414. doi:10.1093/cid/ciaa414.

27. KCDC. https://www.cdc.go.kr/board/board.es?mid=a20507020000\&bid=0019. Accessed $19 \mathrm{Jul}$ 2020.

Figures
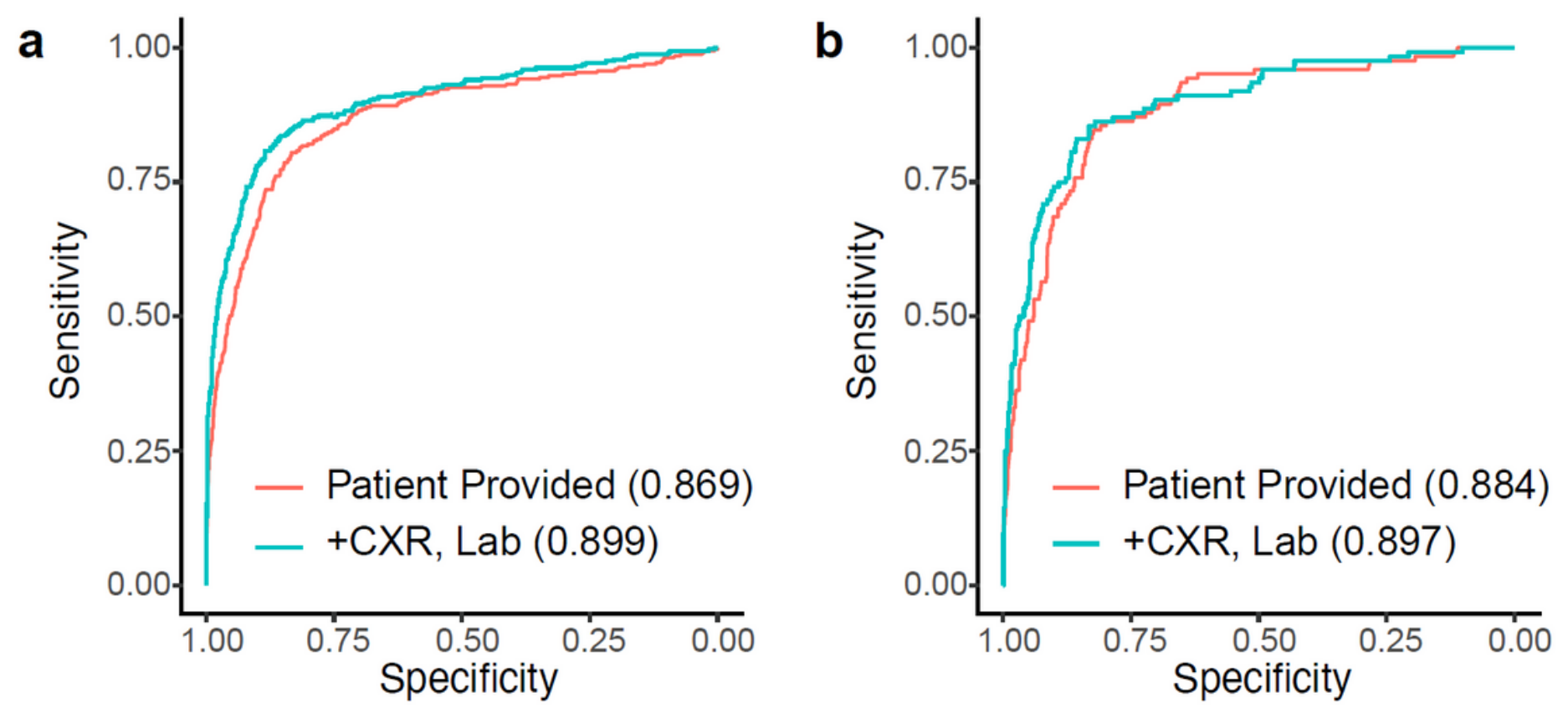

Figure 1

Receiver operating characteristic curves for the A) training and the B) validation dataset. The area under the curves shown in parenthesis. 


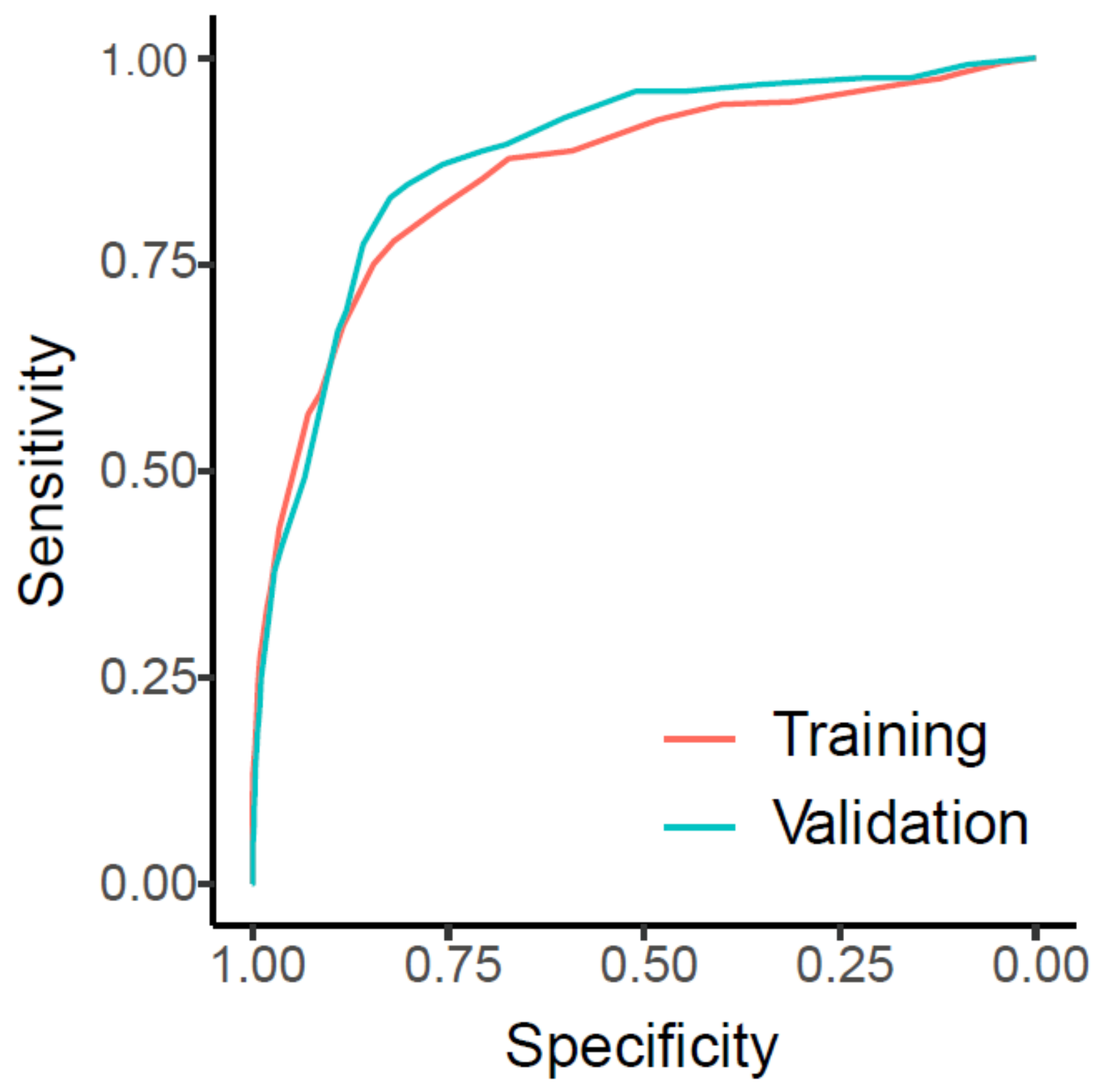

Figure 2

Receiver operating characteristic curve of the newly developed scoring system (COVIC score). Score tested on the validation dataset for predicting patients with COVID-19 requiring ICU care (COVIC score). The area under the curve was 0.880 [95\% $\mathrm{Cl}, 0.847-0.912]$. 


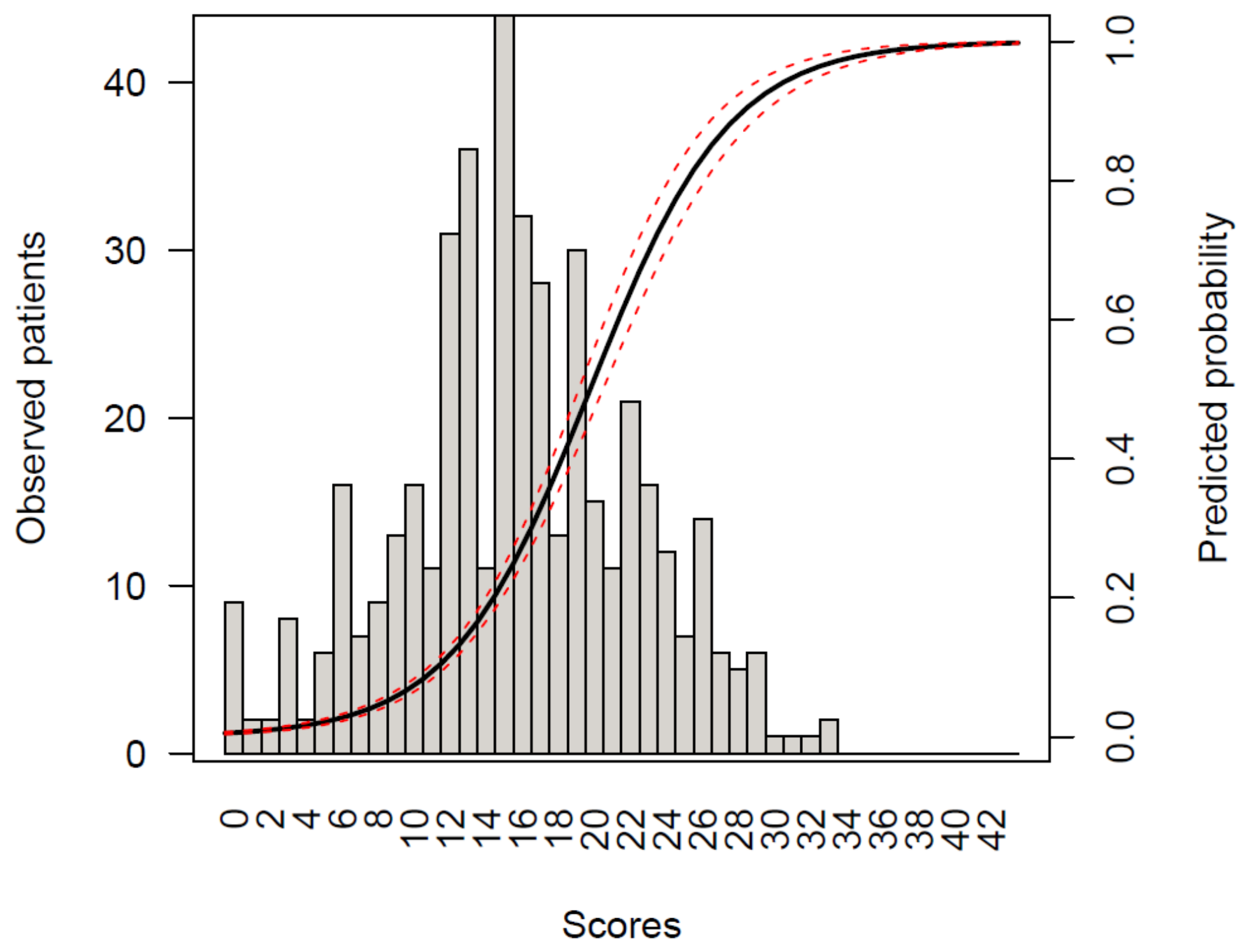

Figure 3

Association between the integer-based score and the probability of the patient requiring intensive care. The red dotted line indicates the $95 \%$ confidence interval. 


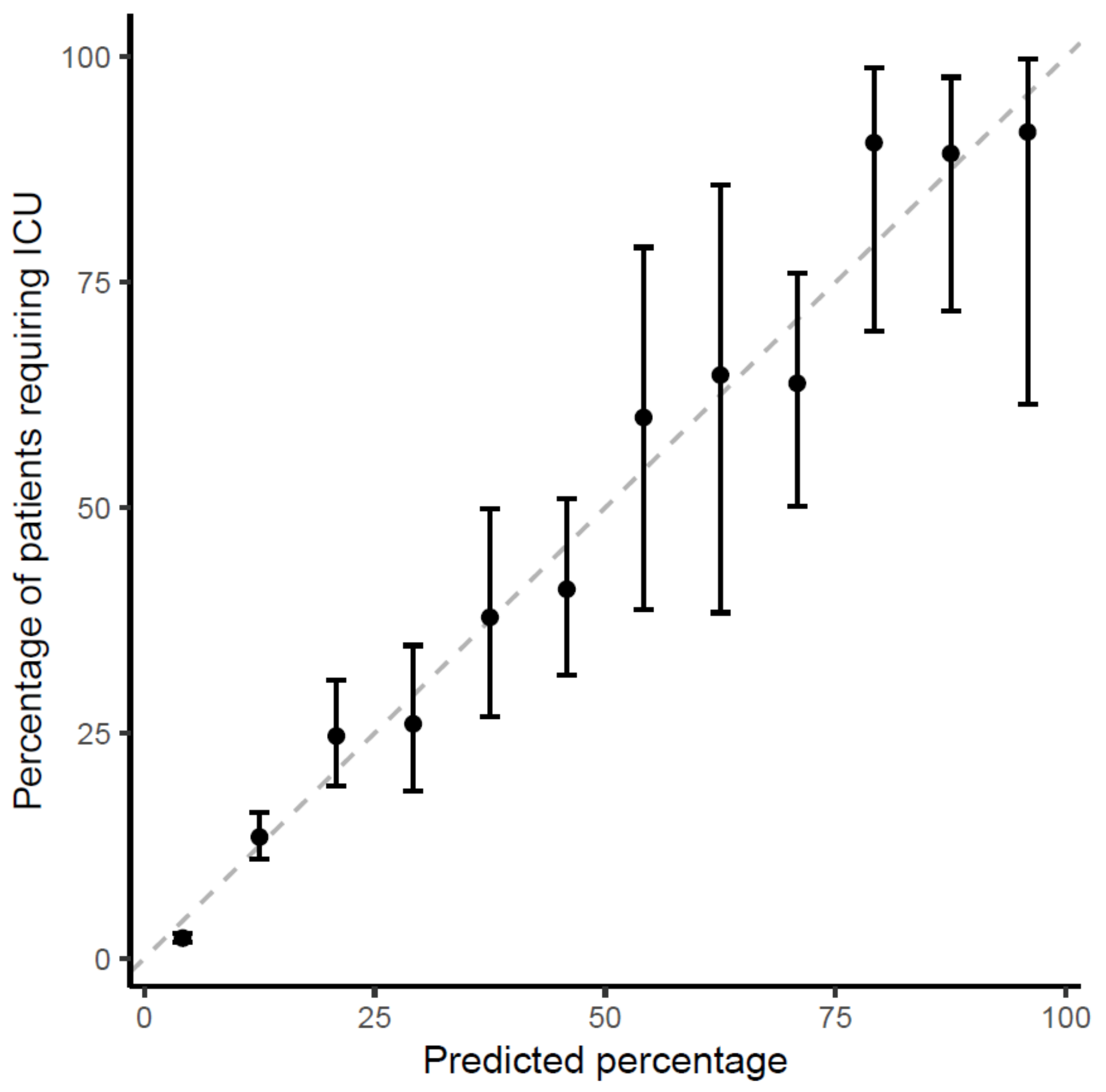

Figure 4

Calibration plot for the integer-based prediction score (COVIC score). 


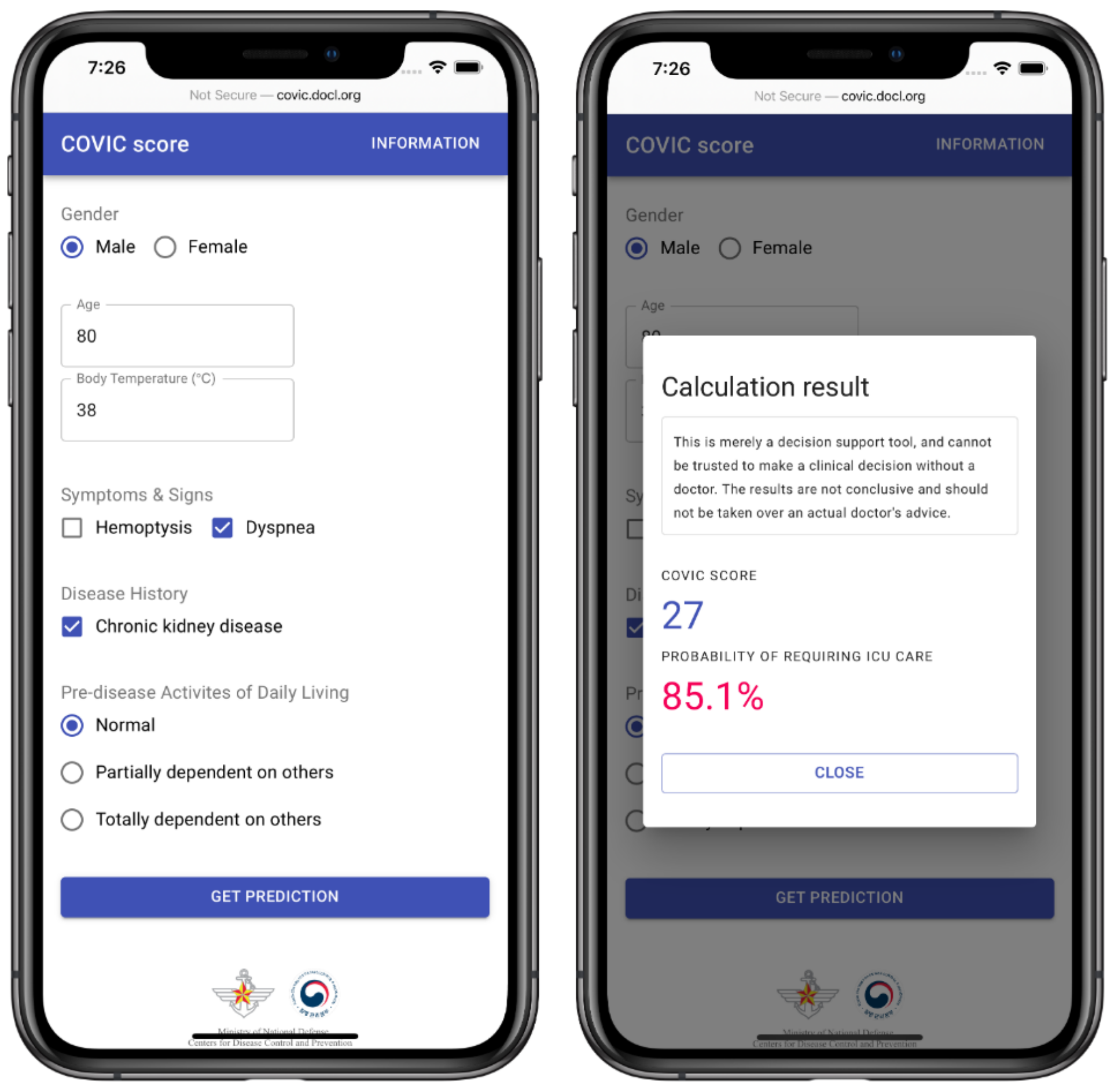

Figure 5

Screenshots of the web application developed for ease of use of the COVIC score. Accessible on the web on a mobile device or a desktop at http://covic.docl.org 